\title{
THE PRINTING OF BACKBONE TITLES ON THIN BOOKS AND MAGAZINES
}

\author{
By P. N. Gould, L. C. Raines, and C. A. Ruckmick
}

To those who have been working in libraries containing magazines in their original covers and bound books of few pages it has probably become noticeable that a very unfortunate lack of uniformity exists in the printing of titles lengthwise on the backs of these magazines and books. While there is in different countries a general disposition to conform to some standard of printing, there is by no means an agreement on the subject in any one country. In general and with many exceptions, the European plan is to print such titles from bottom to top, while the American plan, if there is one, is to print in the reverse direction. A considerable amount of discussion has arisen on the subject, especially in connection with library practice and in the printers' journals. ${ }^{1}$ There are related questions, such as the subscriptions to fullpage plates inserted in reading material, ${ }^{2}$ and the insertion of omitted matter in writing when the insertion is titled in one direction or the other.

Many persons, in discussing the subject, seem to take the view that while they may have individual preferences in the matter they would be willing to set these aside if a universal practice could be established. Libraries insist, for the most part, on the advantages derived from printing titles in the direction from top to bottom, arguing that when magazines are stacked on tables, newsstands, and temporarily on shelves, the logical direction, with the face up, would be from top to bottom, or, when they are in this position, from left to right.

Some preliminary investigations, though crude, have been

${ }^{1}$ American Printer, August 5, 1918. Printing Art, 1918, xxxii, 62. Publishers' Weekly, 1918, xciv, 1763, 1994.

2 Preliminary Report of the Joint Committee on Standards for Graphic Presentation, Amer. Soc. of Mech. Eng., N. Y. 
made on the question, but so far apparently no experimental work has been published. One preliminary study resulted in the decision of seven in favor of printing from bottom up and two in placing the lettering from top down. Three additional answers, however, were noncommittal. In another inquiry an investigator found that among men of his own staff who were preserving pamphlets and frequently writing titles themselves on the backbone, nine out of ten were using the method which involved printing from bottom to top. Three out of four of them declared that any other method was unnatural. On the other hand, so eminent an authority as Melvil Dewey says, "The statement that it has been demonstrated by tests to be easier and more natural to read from bottom to top contradicts all the experiments and investigations I have made for forty years."

Obviously, on further consideration, there are a number of circumstances which are indirectly involved in the question. If ease of reading is to be a criterion, then the way in which the head is more readily inclined undoubtedly has an important bearing. A great number of individuals, from replies received on the subject, indicate that it is easier for them to tilt the head to the left. And, indeed, there is some basis on the side of the musculature of the eyes, neck and shoulders which might favor this inclination. ${ }^{3}$ It is possible, also, that the tilt of the head in writing, being for the most part to the left, has a bearing on the problem. One correspondent, for instance, noted that during the time that he was learning to read and write Hebrew and for some time thereafter his head would habitually tilt to the right instead of to the left. Another authority widely known for his investigations of eye-movement states that he has noticed the invariable practice of right-handed people tilting their penmanship upward, while left-handed individuals habitually write downward.

A relatively large number of individuals have remarked that a differentiation exists between the use of either hand in taking books from the library shelves, the left hand being used to

see the discussion of head-movements relative to Listing's Law in Helmholtz, H., Handbuch der physiologischen Optik, 3rd ed., vol. iii, Hamburg and Leipzig, 1910, pp. 120-123. 
withdraw the book from the shelf while the right is preparing to finger the pages, and in this position the body would be more inclined toward the left. All of these suppositions are conditioned by the height of the shelf above or below the level of the eyes, as well as by the location of the shelf to the right or to the left of the individual. Several replies indicate also that the lighting effect may be a complicating factor. Those who argue in favor of printing from top to bottom seem to be impressed by the analogy of reading printed lines from the top of the page down to the bottom. But it is not unlikely that the analogy is overdrawn and that the factors involved in reading titles of books, when printed at right angles to the usual movement of the eyes, presents a totally different situation from the ocular excursions of ordinary reading.

\title{
Experimental Series
}

Apparatus. The entire problem was taken over as an experimental investigation in the psychology laboratory, and conditions were devised to simulate those of the library. In one of the dark rooms of the laboratory a shelf was designed to be fastened in a number of positions on the wall, being adjusted to heights of four, five and six feet from the floor, these being standard heights in library practice. On the shelf about a score of books were placed and among them a so-called "dummy" was inserted. This dummy measured $26 \mathrm{~cm}$. long by $2 \mathrm{~cm}$. thick. Along its front edge a sliding arrangement was provided into which printed titles could be placed. These titles were printed on heavy white paper and consisted of thirty-eight different names of standard American publications such as:

\author{
THE GEOGRAPHICAL WORLD \\ THE AMERICAN FORESTRY \\ THE AMERICAN WOOL REVIEW \\ DUNN'S INTERNATIONAL REVIEW \\ THE MARINE REVIEW \\ THE CONTEMPORARY REVIEW
}




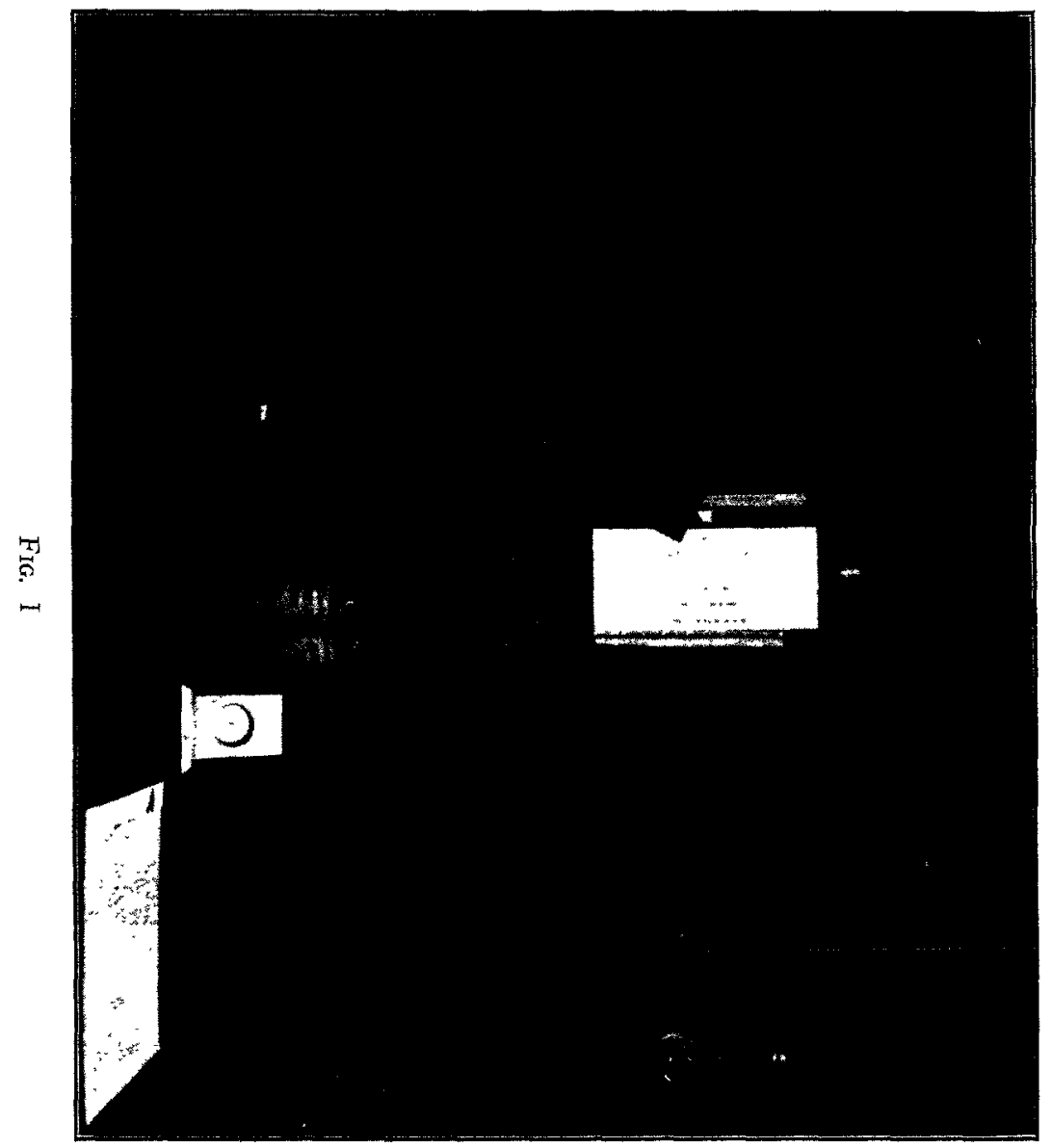


In front of the shelf a double scissors-like shutter was provided which would expose to view the entire length of the "dummy" and some of the neighboring books without suggesting by its own movement the direction to be followed in reading. Later in the experimental series, the "dummy" was replaced by a device that would hold six or more titles at once, the shutter was opened through an arrangement of strings, elastic bands, and pulleys, operated by the experimenter. An electrically operated stopwatch was used to time exposures and reactions of observers. The entire shelf and shutters were painted a dead black and the room was lighted uniformly by means of an artificial "noon-day" light of high power. In another part of the experiment a head rest was provided. The observer was uniformly three feet in front of the title to be read. A pointer was affixed to a wireframe to indicate the approximate place at which the titles were to be read.

Procedure: The experimental investigation began early in Igrg and lasted through the summer of that year. The observers, fourteen in number, were selected from among the advanced classes in experimental psychology. They were given instructions to read aloud as clearly as possible, and at their normal speed, the title that was to be presented to them. Introspections were recorded in about half the cases.

The series were divided, in the first group of experiments, so that $3^{6}$ observations were taken with the titles at the level of the eyes, the same number below, and an equal number above the level of the eyes. It was soon found that the reading of a single title could not be accurately timed under the conditions of the experiment, so, for the most part, cards were made up of from four to eight titles running in the same direction, to be read consecutively, and the resulting time divided by the number of titles read. In the second group of experiments the observers were asked to walk from a spot about five feet to the right or to the left of the shelf and then walk towards the shelf, on the assumption that entrance to the room constantly from one direction would itself influence the observer to favor one side or the other. 
In a final group the observer's head was placed in a head-rest which prevented movement to either side.

In each case, after the observer had taken his position the experimenter would open the shutter and release the stop watch with the same movement. When the reading was completed the watch was electrically stopped. The arrangement of the cards was determined by a chance order but care was taken that each presentation to the left, that is from bottom to top, was at some place matched by a presentation of the same material in the opposite direction.

As a result of our questionnaire (see below) we have also the unpublished records of some class experiments conducted by Professor A. P. Weiss of the Ohio State University and of a few individual experiments performed in the same laboratory. Some disjointed printed material mounted on a board and 200 words in length was presented to the observer to read aloud as rapidly as he could accurately do so. The time was recorded in seconds. Both the copy and the observer were given a variety of positions, viz., (I) the copy upright and the observer upright, material read from left to right; (2) copy upside down, observer erect, material read from left to right; (3) copy turned to the left 90 degrees, observer erect, material read from bottom up; (4) copy turned to the right 90 degrees, observer erect, material read from top down; (5) copy turned to the left 90 degrees, observer bending to the left 90 degrees, material read from bottom to top; $(6)$ copy turned to the left 90 degrees, observer bending to the right 90 degrees, material read from bottom to top; (7) copy turned to the right 90 degrees, observer bending to the left 90 degrees, material read from top to bottom; (8) copy turned to the right 90 degrees, observer to the right 90 degrees, material read from top to bottom.

The time values in these series do not present significant differences. For example, there is a difference of only two seconds in the median values of series 5 and 8 , which series approximate most closely the question raised in our problem. It is significant, however, that out of fourteen observers who expressed a preference for one of the last four positions, eleven preferred position 5 , two preferred position 8 , and one was doubtful regarding 5 or 8 . This apparently gives a favorable vote to the position which involves bending to the left and reading material from bottom to top.

Observations were also taken by five persons, in the library, to ascertain which way the head and body are bent when reading book titles in the stacks. In 187 cases observed, 29 per cent. bent voluntarily to the right, while 7I per cent. bent voluntarily to the left. Obviously these results should be suitably qualified by reason of the fact that they were not obtained under experimental conditions. Such factors as differences in illumination, position of books as regards the aisles, just previous position of the observer, height of books from floor and similar circumstances, clues to which could 
very likely be obtained in individual reports, were not taken into account. The figures speak for themselves only in the mass. And yet, of course, when so taken they are very good indicators of a possible conclusion.

Results: As far as our own statistical records go, the time values and also the number of times which indicated a faster reading for one or the other direction show no general significant features. The reading times indicate the least variation when comparisons are made in the values obtained in reading upwards with those obtained in reading downward. There is, of course, large individual variation with the observers. The only figures that seem to have any relevancy are the total times that the series was read faster in one direction than in the other, and there, only as regards that phase of the problem which took into account the height of the shelf from the floor and the direction from which the shelf was approached. When the shelf is above the level of the eyes the bottom to top direction is most favorable, whether it is approached from the right or the left, although slightly more so when approached from the right. In 69 per cent. of the cases the times were faster from bottom to top and in $3 \mathrm{I}$ per cent. they were faster in the reverse direction. When these percentages are factored into values which signify the direction from which the shelf was approached, we find that the value for the approach from the observer's right reaches as high as 77 per cent., whereas that for the approach from the observer's left decreases to 59 per cent. At the level of the eyes there seems to be no appreciable difference in time values or in the number of times either direction is read faster. This is markedly true in the series in which the head was fixed. In the series in which the shelf was placed below the level of the eyes, there is a slight preference for the top-to-bottom direction as expressed in the number of times this direction was read faster. The percentages are: .57 for the top-to-bottom and .43 for the reverse direction. When these values are factored into figures which indicate the direction of approach, they increase so that the topto-bottom direction is .64 when approached from the right and .49 when approached from the left. The writers, however, wish 
to emphasize the fact that there is so slight and sometimes so inconsistent a difference in the actual time values for reading in one or the other direction, that comparatively little evidence in favor of printing titles uniformly either from bottom to top or from top to bottom is forthcoming from the mere statistical account of our investigations and more stress is to be placed on the observers' comments. Stated in other words, as a matter of sheer efficiency gauged in the time actually spent reading the titles, we find no noteworthy or uniform difference.

Something must be said, nevertheless, both for the mental attitude of the reader as regards his reaction to the confusion which now obtains respecting the printers' and publishers' practice and for the time that may actually be lost before the reading of the title is begun. This latter value was, of course, omitted entirely from our calculations. It is a value hard to obtain, and compares with the well-known "fore-period" of the reactiontime experiments.

The introspective comments in almost every case diclose prominent kinaesthetic sensations localized about the head and neck and occasionally in some of the larger trunk muscles. These appear before the reading is begun and last through the reading period. Frequently they are affectively colored, and unpleasantly so before a sudden change of position, e.g., when the direction of reading is opposite to that anticipated. To quote:

"Slight tendency to tilt head according to reading of card. Tendency to read from bottom to top of card. Cards blurred more or less by fixed position-eyes used to slight tilting of head to ease strain of reading."

"Pleasantly colored sensations while waiting for opening of shutter-desire to begin quickly. Unpleasant emotion when top of sheet is at the right, pleasant when at left (bottom to top)."

"When the cards were perceived to read bottom to top, there was a kinaesthetic sensation in the neck resulting from turning the head to the left side."

"When cards were shown there was an involuntary kinaesthetic twitch in the muscles of the eyes toward the upper right-hand corner in all cases."

"When the cards were exposed there was a kinaesthetic sen- 
sation of movement of my eyes to the lower left-hand corner of the cards. If I perceived that this was the point at which it was easiest to begin reading (if the lines ran upward) I began reading at once without difficulty. In case they ran downward, however, it was necessary to focus my eyes at the upper right-hand corner."

It is clearly seen that the mental accompaniments of the reading must be taken into account. They parallel the actual reading in many cases without disturbing the reading time. In most cases, however, there are kinaesthetic adjustments made in anticipation of the reading and if these are later to be altered an unpleasant affection results which seldom disturbs the reading. It is barely possible that if the experiments were to be continued and the time accurately measured from the opening of the shutter to the beginning of the reading, especially if the reading were done through an electrically controlled lip-key or the Dunlap voice-key, significant time values would result for the two directions.

\section{Questionary Series}

While the investigation was proceeding, some 92 answers to questionnaires were received, about one-half of this number from colleagues on the faculty of the University, and the other half from psychologists selected from the American Psychological Association. A copy of this questionnaire follows.

Dear Sir:

Librarians and others are just now discussing the problem of printing titles lengthwise on the backs of periodicals and thin books. Some maintain that the more readable direction is from top to bottom; others take the opposite view. The question has been referred to us for experimentation and the investigation is now under way.

In connection with the experimental results that we are obtaining, it is of interest to us to learn the personal opinions of those who are accustomed to the use of such material. For this reason we should very much like an expression of your reaction to the following statements:

(I) When publications of this sort are in position on a book-shelf among other miscellaneous books, $I$ find that $I$ am inclined to read the title more readily when printed from $\{$ top to bottom

\{ bottom to top

(2) There are circumstances, however, which may modify this inclination; these are: 
(3) When inscribing titles along the backbone of reprints inserted in covers, I usually write them from $\left\{\begin{array}{l}\text { top to bottom } \\ \text { bottom to top }\end{array}\right.$

(4) I am normally $\left\{\begin{array}{l}\text { right-handed } \\ \text { left-handed }\end{array}\right.$

A number of affiliated questions are frequently raised in this connection. Among them are: (a) Is the head generally inclined more readily to one side than the other or are there frequent variations? (b) Does the predisposition to read lines from left to right and from top to bottom affect the question raised? If you care to discuss these questions or related questions on the back of this sheet in addition to your answers to previous questions, we should be glad to hear from you.

Thanking you for your coöperation, I am

Very truly yours,

(Signed) Christian A. Ruckmick.

When the votes are counted without reservations we find 48 in favor of reading from bottom to top and 34 in favor of the reverse direction. Fifty-three were in favor of writing the titles on the backbones of monographs from bottom to top, and 30 in the reverse direction. In this connection it is interesting to note that while some indicate a preference in reading from top to bottom they state in reply to the third question that they write in the titles in the reverse direction. On the other hand, not a single case appears in which the bottom-to-top method of reading is combined with the habit of writing titles from top to bottom.

If space permitted it would be instructive to publish all of the individual comments made in reply to questionnaires. Some have already been summarized in our introduction to the problem. There seems to be without question an individual difference in the matter, with the majority of habits formed in favor of tilting the head to the left, making the bottom-to-top reading probably the more agreeable one. At best many consider the practice a necessary evil and, as one well-known psychologist has it, one would "vastly prefer any kind of makeshift in the way of horizontal abbreviation which would obviate the necessity of straining one's eyes to the required position in order to read the vertical type."

Summary and Conclusion: It will be seen from our studies that, so far as the rapidity of reading is concerned, there seems 
to be no pronounced difference in favor of one direction or the other. With certain factors added, such as height of shelf, position of the book to the right or to the left of the observer, individual habits, and above all, individual preferences, especially as these are emphasized in our verbal reports, then there appears a significant margin of choice in favor of the bottom-to-top direction. Further experiments investigating the period just before reading and possibly also the period after reading should be planned and introspections recorded.

\section{Replies to The Questionary}

Since the comments, theories, and explanations that were returned with the printed questionnaires would be lost in any statistical treatment of the results obtained, it is better to let them tell their own story without modification. Accordingly there is appended a series of such discussions selected from the entire set of returns. Only those have been omitted whose statement of the point is found in some other quotation and is usually therein better expressed. Many of the statements come from prominent psychologists and other widely known men of science; but because it is doubtful whether we could properly attach their signatures to material thus obtained, we are presenting them only on their intrinsic merits.

"I try to have the legend on the 'backbone' of every publication that is not wide enough to print across printed to read from top to bottom. As my function is more that of the maker of the periodical than of the user or the librarian, I am interested in having all such arrangements meet the convenience of the persons who use them.

The best summary of the argument for top-to-bottom readings is found in H. P. Ward's "American College Catalog," page 79:

"Although some college catalogs are made with the backbone title reading from bottom to top, many are properly printed with the title reading from top to bottom. The reason for this form is that when the catalog is laid flat on a table or desk, front side up, and other catalogs are placed on top of it, it is possible to read the backbone title without taking it out of the pile. The best explanation of this matter would be a glance at a pile of old magazines. It would be far better if the few catalogs whose backbone titles read from bottom to top could be treated as the great majority are, with the backbone title reading top to bottom, for the sake of uniformity of appearance in the Exchange Catalog Shelves of hundreds of institutions and libraries. The librarian customarily passes along a shelf from left to right. reading from top to bottom. He is entitled to some consideration.

It seems to me that the question of uniformity is the important one here." 
"When I started in to print the Review I was perfectly shocked to find that the printer had printed the title on the back of the magazine from bottom to top. It looked all wrong to me and yet the printer declared that that was the usual custom. I told the usual custom to be hanged; the Review was going to suit my aesthetic taste, and so it follows it from top to bottom."

"(a) I find I incline my head more readily to the left than to the right. (b) Predisposition to read from left to right favors printing titles from the top toward the bottom because when the book is laid flat on the table or book-shelf with the front cover up, the printing on the back is then rightside up and reads from left to right. Perhaps the disposition to read from top to bottom also favors the printing of the title in this way.

In spite of the considerations mentioned in (b), I prefer the reading from bottom to top. In this connection, it may be worth while pointing out that this is the practice of the government printing office-at least it is so in such bulletins as fall under my eye as I write this."

"I return herewith your outline and in this connection wish to say that $I$ have gone over my private library including many thousand pamphlets and as nearly as one can determine by a rapid examination the following is true of the publications which have printed labels on the backbone. Older journals printed in America have the label running from bottom to top, where the same periodical in the last dozen years has changed its habit. The same is true of government publications issued in the Philippines. However, those printed in Washington, namely the Geological Survey and the United States National Museum, have consistently followed the rule of beginning the line at the bottom and printing towards the top. All of the English periodicals to which I referred have the titles on the backbone started at the bottom and reading towards the top. All recent French (one exception), German (one exception), Italian and Russian publications make the title read from the bottom towards the top. In old German publications this seems to have been the exceptional method and of the few I had almost all had the title printed in the opposite direction."

"Recent scientific publications of medical character printed in this country have the backbone title reading from the top towards the bottom."

"I found on attempting to study the shelves, I approached them always in such a way as to stand at the right and then it was natural to read from the bottom towards the top following direction from left to right. When I tried to stand at the left in which it was possible to read easily from the top to the bottom, it required considerable effort to push my head into the proper position and it was not natural to handle the books because in separating them one always looked at the back of the pamphlet, whereas in approaching from the right one could pull the books apart and the eye fell naturally on the front of the pamphlet. It seemed to me that there was not any difficulty in inclining the head towards one side rather than towards the other. I am not sure that habit is the factor involved but my experiment lead to the distinct feeling that one way was natural and the other not." 
"In a filing drawer where pamphlets are grouped back up, it is very inconvenient to have the title read from top to bottom. Come over to see my library some day."

"I believe this habit of reading from below upwards may be connected with that of writing with the paper tipped upward at the right. It would be very awkward to write with paper tipped downward at the right; that would demand a cramped inward movement of arm, whereas inclining paper upward at right gives a free outward movement of arm."

"In reading the titles of volumes on shelves printed lengthwise of the volume I always stand on the left side of the volume and the head is inclined to the right side. I find it difficult to stand on the right side and to incline the head to the left. All geological publications of United States and Canada, British colonies and nearly all British print from bottom to top. Perhaps this may be reason for my preference."

"It would be inconvenient to write from bottom to top as I hold book in left hand and it requires less energy to turn it into position to write bottom-top than top-bottom."

"The 'psychology' of it with me appears to be that when a lot of such things are shelved in alphabetical order by author (or title) I seem to be reading them down the pile,--much as I would continue the alphabet down onto another shelf; so they resemble a series of alphabetical titles on a printed paper which I read from top to bottom far more readily than I should if they began at the bottom with $A$ and wound up with $Z$ on the top line."

"All narrow columns such as the headings to columns in tables read from top to bottom. This holds true even though the headings are spelled out by single letters. The habit is thus formed of reading downwards. This may seem inconsistent with the common practice of placing tables and cuts that run lengthwise of the page so they read from the bottom as the left hand side of the page. But to read such tables we must turn the book, and the most natural method is to turn it "clock-wise."

"My head inclines more readily to the left. I attribute this to the fact that I always have the light come over my left shoulder, and hence usually read with head inclined a little to the left. Likewise, right-handed people write with heads bent to the left."

"I find also that I have had to put labels on a very considerable number of small slim botles and in every case it reads from bottom to top. I naturally pick the bottle up in my right hand by the top and swing the bottom to the left, so the bottom to top label is easily read. I have counted 20 booklets and pamphlets here which have titles lengthwise on the back-all as prepared by the printer. Eight have titles bottom to top and 12 top to bottom. To my mind there is little difference after we get used to either, but uniformity is desirable."

"Titles of books are usually printed at the top, often back of the volume. 
The beginning of the lengthwise title should be near the same line as the cross-wise title."

"In searching for books on a shelf, using titles, a man usually works from left to right, and so likes to catch the clue word of a 'back-bone' title on the same level as other titles, without fumbling lower down."

"In my own case, I am quite positive in this matter. Even in so small a matter as inserting a word I write it tilted of books you naturally follow, instead of backing along, as you cannot do when you read from the bottom up."

"Personally $I$ have a slight preference to turn my head to the left in looking at such titles which would mean printing from bottom to top. I arrange MSS. in folders on that principle. I am right-handed. On the other hand, I am strongly of the opinion that all such titles should be printed from top to bottom for the following reason. Unbound magazines are more frequently laid flat than on edge. It is absurd to lay the magazine face down; and it looks equally foolish to see the title upside down on the back. The edge titles of unbound magazines should therefore be printed from top to bottom. Why have one rule for magazines, another for books? A thin book is often laid flat and it is much simpler to have the title page uppermost and the back title upright. On the ground of simplicity I should recommend very urgently that one uniform rule be adopted, namely, top to bottom."

"The only justification, in my opinion, for the bottom to top method of printing titles is the stacking of books and reprints horizontally, rather than vertically, and even this is doubtful value. For, when books are kept horizontal, the book to be consulted must be turned over through an angle of $90^{\circ}$ before the title page is readable."

"A right-handed person commonly writes with the lines sloping from lower left to upper right of the visual field to accommodate his forearm movements. Never the opposite slope as far as $I$ have observed. This is reversed for left-handed persons. This gives a considerably greater familiarity to upward reading lines to the majority of persons. My practice is, wherever I am not otherwise constrained by positions on a ladder or other considerations, to turn pages with the right hand and hold a book with the left. This naturally leads to taking a book from the shelves with the left hand. As a matter of economy of effect this is worth encouraging by upward reading titles. The main point is uniformity. We can readily adapt ourselves to either way of printing. Lack of uniformity is disturbing and fatiguing."

"If I incline, not only in order to read, but read and handle at the same time, I incline to the left because I handle with the skillful right and support the body with the unskilled left. The conditions are so complex, that it is of little average advantage to use one method of writing titles rather than the other. We might as well decide it by tossing up a coin. But it is 
of the greatest advantage to have all titles written the same way. Maybe one style is already preponderant. Then that should be generally adopted."

"If a book is lying before you, right side up, opening at right, a title printed lengthwise on back can be read by moving head and tipping to left, with a slight raising of the back of book, if title is printed from bottom to top. But if printed from top to bottom, greater movements (changes of position) are required. This seems to me to be the only fact indicating which is the 'normal' method of printing. Either method would be satisfactory, if made universal. One would as easily form the habit of looking one way as the other along the shelf. I prefer, however the 'normal' arrangement of reading from bottom to top."

"If I were sitting in front of a bookcase at some distance I would prefer that the titles be printed from top to bottom. If I were in front of a bookcase and passing from right to left, I would prefer that the titles be printed from bottom to top. If I were to pass from left to right I would prefer that the titles be printed from top to bottom. I would naturally pass from right to left. If $I$ were sorting through a pile of reprints placed in front of me, top cover up, I would prefer that the titles be printed from bottom to top."

"In reply to your inquiries of February I2th, I write to say that the practice to which you refer always fills me with suppressed rage, for unless the book concerned happens to be at the level of my eyes, $I$ find myself attempting to stand on my head in order to compass the necessary reading. My impression has been that I find this process less distressing when the title is entered from top to bottom than when in the reverse form; but either practice has always seemed to me an invention of the Evil One. I vastly prefer any kind of makeshift in the way of horizontal abbreviation which will obviate the necessity of straining one's eyes to the required position in order to read the vertical type. Although $I$ have never made careful experimental study of the matter, my general impression has been that if books were on shelves below the level of my waist, I would prefer to have the titles run from the bottom up. With the shelving in my own library this occurs less frequently than the converse way with the books above my waist and perhaps explains my supposed preference for the title running from the top downward."

"My head inclines more easily and automatically toward the left. I read more comfortably with a left-hand inclination. The usual flat position of a book held in front of the face, title page up, brings the reading on the back from bottom to top."

"I slightly prefer inclination of the head to the left, but I am inclined on the other hand to approach a book shelf from the left and this necessitates the reading of titles from top to bottom. Undoubtedly, the habit of reading from left to right affects the situation. I recently noted that when titles of books are printed lengthwise of a page, the orientation differs. There should certainly be uniformity of practice in this connection. I pre- 
fer to have the lines begin at the bottom of the volume extending thus from bottom to top. Evidently the conditions which influence me in this case are somewhat different from those in the case of the title on book or volume. If instead of reading titles on a book shelf, I lift a volume from the table to read lengthwise title, I very much prefer to read from bottom to top."

"In reply to your questionnaire with regard to printed titles on the backs of books and periodicals I beg leave to say that I find on examining my preferences that I prefer a title that reads from the bottom to the top. I find that my method of approaching the bookcase is such that I naturally tip my head toward my own left hand and stand in such a position that I read up rather than otherwise. I did not realize that I had the preference as fully as I find that I have. I am quite right-handed and do not know of any general considerations that would have prompted the development of this habit. I call your attention to one fact, well known to oculists; there is a tendency for the head to be bent so as to bring the plane of normal fixation of the two eyes into a position where there will be the least muscular strain on the eyes. The neck muscles in this way coördinate in making eye adjustment." 